\title{
CORRESPONDENCE
}

\section{Rethinking relationships between natural products}

\section{To the editor:}

Glycobiologists often describe oligosaccharides as a third class of macromolecules, elevating them to a status otherwise reserved for nucleic acids and proteins. However, striking chemical and biological similarities suggest that a more meaningful conceptual kinship exists between oligosaccharides and terpenes.

Small molecules have been highlighted in Commentaries in Nature Chemical Biology ${ }^{1}$, including as orphans of the macromoleculefocused central dogma ${ }^{2}$. So it was of interest that Peter Seeberger described oligosaccharides as comprising the third class of macromolecules, together with nucleic acids and proteins, in an Essay in Nature ${ }^{3}$. Seeberger is not alone in this conceptualization; it is arguably a centerpiece of glycobiology lore. In my own experience, this conceptualization made its way into my teaching of organic chemistry and chemical biology to students.

But does it make sense, either chemically or biologically? I argue that it doesn't, and I offer an alternative conceptualization: oligosaccharides should be viewed in the same light as the small-molecule terpenes, not as another class of macromolecules. Today when I teach my students, I ask them to consider the following observations:

(1) Like small molecules, oligosaccharides are primarily involved in dynamic information flow, in contrast to the heritable information flow mediated by nucleic acids and proteins.

(2) Nucleic acids and proteins are the products of template-mediated synthesis in cells, and as a result they are directly linked to (and are the mediators of) the heritable information encoded within genomes. Oligosaccharides, like naturally occurring small molecules, are synthesized by conventional, non-template-mediated synthesis in cells, and therefore they are less directly linked to information encoded in genomes ${ }^{1}$.

(3) Striking similarities exist between the oligosaccharides and terpenes, in terms of both their biosynthesis and cellular functions: (i) the biosyntheses of oligosaccharides and
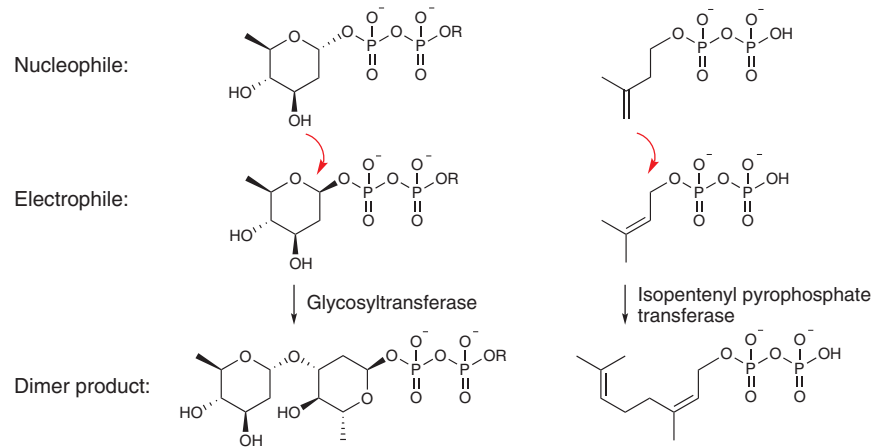

Figure 1 Oligosaccharides and terpenes are biosynthesized using conceptually similar strategies that do not involve the template-mediated synthesis that is characteristic of nucleic acid and protein biosyntheses. Oligosaccharides are on the left (dideoxyglucose is shown for illustration) and terpenes are on the right. Cells use transferase enzymes to synthesize oligosaccharides and terpenes (glycosyltransferases versus isopentenyl pyrophosphate transferases; illustrated) and tailoring enzymes to modify nascent oligosaccharides and terpenes (not illustrated). Many glycosyltransferases use nucleotide diphosphates ( $\mathrm{R}=$ nucleotide) as indicated, although others use nucleotide monophosphates.

terpenes both involve transferase-catalyzed, bimolecular displacement reactions of fiveor six-carbon monomer building blocks that contain both nucleophilic groups and electrophilic diphosphates. These reactions occur iteratively, yielding polymers that are typically dimers to hexamers (Fig. 1). (ii) These biosyntheses next involve tailoring enzymes that modify the oligomers (dimers to hexamers), yielding products that have greater structural complexity and diversity (see Fischbach and Clardy, p. 353). Tailoring reactions include alkylations, acylations (for example, lipidations), carbamoylations, sulfonylations, oxidations, reductions, macrocyclizations (including transannular cyclizations) and "trimming reactions." The resulting oligosaccharide and terpene products have similar sizes and ranges of molecular weights, which are substantially diminished relative to natural nucleic acids and proteins. (iii) In some cases, additional transferase enzymes attach the resulting products to proteins, altering the proteins' functions by, for example, anchoring them to the inner leaflet of the plasma membrane. Transferasemediated attachment of the terpenes farnesyl pyrophosphate or geranylgeranyl pyrophosphate to Ras proteins yields farnesylated and geranylgeranylated proteins. The activities of such proteins are altered by their terpenemediated association with the inner leaflet. Likewise, transferase-mediated attachment of glycosylated lipids to otherwise cytosolic proteins is a common cellular mechanism for inducing such proteins to associate with the inner leaflet.

Conceptualizing oligosaccharides as kins of terpenes and other small molecules rather than as kins of nucleic acids and proteins may bring greater clarity to students and researchers when they study this area of chemical biology.

\section{ACKNOWLEDGMENTS}

I thank colleagues at the Broad Institute of Harvard and MIT, Stanford University, and the Harvard University Department of Chemistry and Chemical Biology_especially J. Clardy, D. Kahne and

G. Crabtree-for their insights and encouragement.

\section{COMPETING INTERESTS STATEMENT}

The author declares no competing financial interests.

\section{Stuart L Schreiber}

The Howard Hughes Medical Institute, Broad Institute of Harvard and MIT, and the Department of Chemistry and Chemical Biology, Harvard University, 7 Cambridge Center, Cambridge, Massachusetts 02142, USA.

e-mail: stuart_schreiber@harvard.edu

1. Walsh, C.T. Nat. Chem. Biol. 1, 122-124 (2005).

2. Schreiber, S.L. Nat. Chem. Biol. 1, 64-66 (2005)

3. Seeberger, P.H. Nature 437, 1239 (2005). 